\title{
Measurement of the inelastic neutron scattering cross section of ${ }^{56} \mathrm{Fe}$
}

\author{
R. Beyer ${ }^{1}$, E. Birgersson ${ }^{1}$, A. Ferrari ${ }^{1}$, D. Gehre ${ }^{1,2}$, E. Grosse ${ }^{1,2}$, R. Hannaske $^{1}$, A.R. Junghans ${ }^{1, \text { a }}$, \\ R. Massarczyk ${ }^{1}$, A. Matic ${ }^{1}$, R. Nolte ${ }^{3}$, R. Schwengner ${ }^{1}$, and A. Wagner ${ }^{1}$ \\ 1 Forschungszentrum Dresden-Rossendorf, Postfach 5101 19, 01314 Dresden, Germany \\ 2 Technische Universität Dresden, Institut für Kern- und Teilchenphysik, 01062 Dresden, Germany \\ 3 Physikalisch-Technische Bundesanstalt, 38116 Braunschweig, Germany
}

\begin{abstract}
At the superconducting electron linear accelerator ELBE at Forschungszentrum Dresden-Rossendorf the neutron time-of-flight facility nELBE has become operational. Fast neutrons in the energy range from $200 \mathrm{keV}$ to $10 \mathrm{MeV}$ are produced by the pulsed electron beam from ELBE impinging on a liquid lead circuit as a radiator. The short beam pulses of $10 \mathrm{ps}$ provide the basis for an excellent time resolution for neutron timeof-flight experiments, giving an energy resolution of about $<1 \%$ at $1 \mathrm{MeV}$ with a short flight path of $5 \mathrm{~m}$. By means of a "double-time-of-flight" setup the (n,nâ) cross section to the first excited state of ${ }^{56} \mathrm{Fe}$ has been measured over the whole energy range without knowledge about cross sections of higher-lying levels. Plastic scintillators were used to detect the inelastically scattered neutron and $\mathrm{BaF}_{2}$ detectors to detect the correlated $\gamma$-ray.
\end{abstract}

\section{Introduction}

Partitioning of nuclear waste and transmutation of long-lived isotopes to nuclides with shorter lifetime is an important topic in international research to provide sustainable and green-house gas emission free sources of energy. The Generation IV International Forum has selected six nuclear energy systems for which research and development are ongoing to confirm their viability and to demonstrate their expected performance that includes the objective of producing less waste. In the considerations for waste reduction the possible use of fast (i.e. un-moderated) neutrons as coming directly from the fission process is of great importance as most of the proposed systems use a fast neutron spectrum. Reliable predictions of the relevant physical processes and the optimization of the related facilities depend on the availability of high-quality nuclear data. The data needs have been investigated by the Working Party on International Nuclear Data Evaluation Co-operation of the OECD Nuclear Energy Agency. Two important research fields were identified to be the inelastic scattering of fast neutrons on structure materials in reactors and transmutation devices and the neutron induced fission process of minor actinide nuclei. At Forschungszentrum Dresden-Rossendorf the first compact photoneutron source at a superconducting electron accelerator dedicated to measurements in the fast neutron range has been built. There is also a project at KAERI, Republic of Korea, to install a compact photoneutron source at their electron accelerator [1].

\section{Photoproduction of neutrons at nELBE}

As the neutron spectrum originating from the nuclear photo effect resembles very much the fission neutron spectrum a high intensity electron beam allows suitable measurements in the fast neutron

\footnotetext{
a e-mail: a.junghans@fzd.de

This is an Open Access article distributed under the terms of the Creative Commons Attribution-Noncommercial License 3.0, which permits unrestricted use, distribution, and reproduction in any noncommercial medium, provided the original work is properly cited.
} 


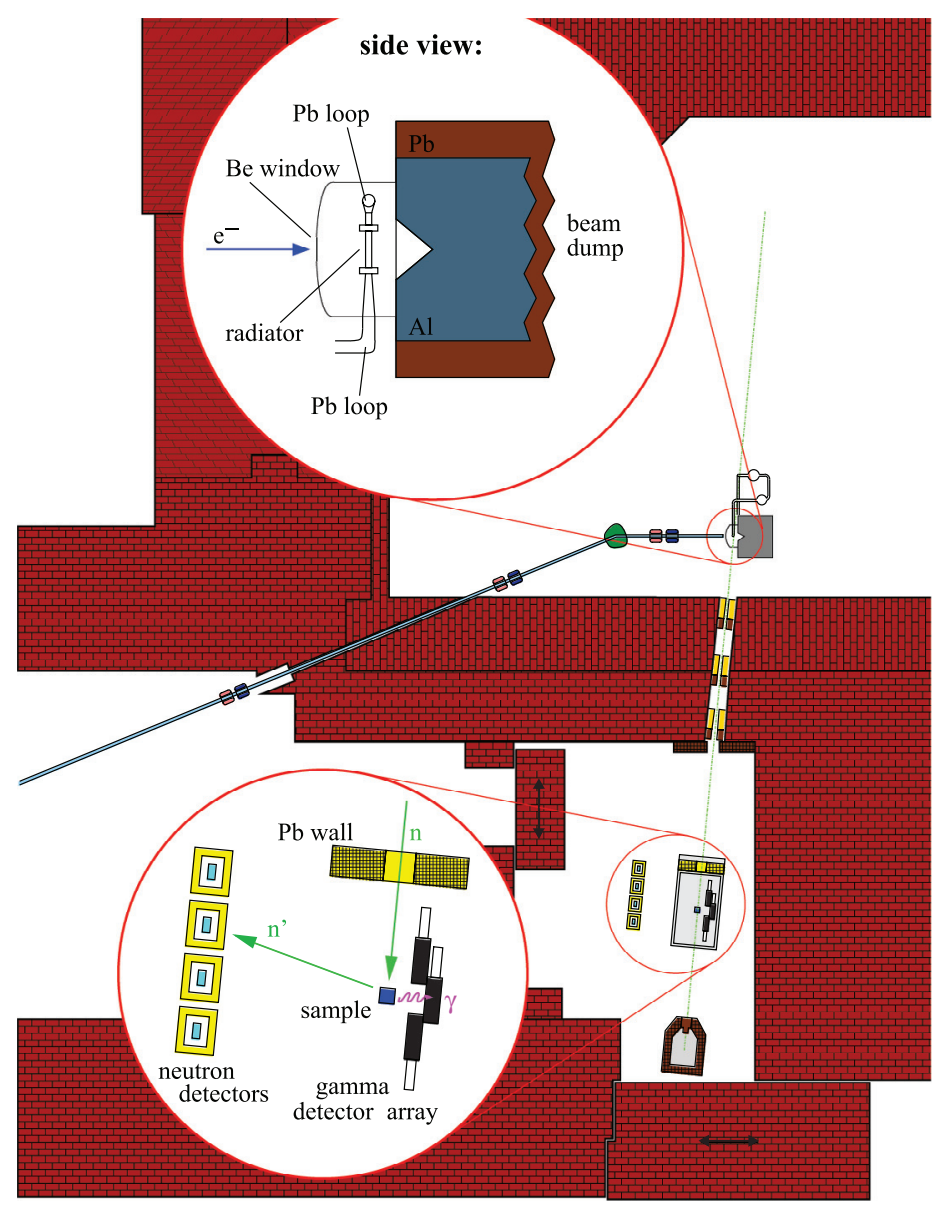

Fig. 1. Floorplan of the nELBE photo neutron source. The inset in the upper left shows a cross section of the liquid lead circuit. The neutron beam is formed by collimator made from sections of lead and borated polyethylene. The experimental setup is reached after a flight path of ca. 5-6 $\mathrm{m}$.

domain. The neutrons are generated by $(\gamma, n)$ reactions with bremsstrahlung from the high intensity electron beam. At the radiation source ELBE of the Forschungszentrum Dresden-Rossendorf (FZD) electrons are accelerated up to $40 \mathrm{MeV}$ in cw-mode by superconducting rf-cavities. The maximum average beam current at a micropulse rate of $13 \mathrm{MHz}$ is $1 \mathrm{~mA}$. The neutron source strength at the nominal beam current has been calculated with MCNP-4C3 to be $10^{13}$ neutrons/s. The accelerator can produce high brilliance beams with variable time micropulse repetition rate and duty cycles. The bunch length is smaller than $10 \mathrm{ps,} \mathrm{so} \mathrm{that} \mathrm{the} \mathrm{time-of-flight} \mathrm{resolution} \mathrm{is} \mathrm{not} \mathrm{degraded} \mathrm{and} \mathrm{short}$ flight paths can be used with a high resolution detection system. At $\mathrm{E}_{n} \approx 1 \mathrm{MeV}$ a resolution $\Delta \mathrm{E} / \mathrm{E} \approx 1 \%$ can be reached with detectors of about $1 \mathrm{~ns}$ resolution. Time-of-flight measurements take advantage of a high pulse repetition rate of $100 \mathrm{kHz}$ to $500 \mathrm{kHz}$. This is nearly a factor of 1000 higher than the pulsed operation at normal-conducting accelerators. The instantaneous neutron and photon flux is lower, which can lead to improved background conditions from the scattered photon flash. At ELBE a superconducting RF injector has been developed [2] that will allow to reach a bunch charge of up to $1 \mathrm{nC}$. With a repetition rate of $500 \mathrm{kHz}$ this corresponds to an average current of up to $0.5 \mathrm{~mA}$.

The neutron flux at ELBE is limited by the maximum beam power acceptable on the neutron producing target and not by the available beam current from the accelerator. A liquid lead circuit is used to cope with the very high beam power deposition $(\mathrm{P} \approx 5 \mathrm{~kW} / \mathrm{g})[3]$, see Fig. 1 . The electron beam passes through a beryllium window mounted on a stainless-steel vacuum chamber and hits the radiator, consisting of a molybdenum channel confining the liquid lead. The channel has a rhombic cross 
section with $11 \mathrm{~mm}$ side length. The electrons generate bremsstrahlung photons which in secondary $(\gamma, \mathrm{n})$ reactions on lead release neutrons. These leave the radiator almost isotropically, while the electrons and photons are forward-peaked. The collimator of $2.6 \mathrm{~m}$ length contains three replaceable elements of lead and borated polyethylene that are mounted inside a precision steel tube [4]. Background radiation through the concrete door to the left of the experimental setup in Fig. 1 has been minimized by additional concrete shielding and geometric realigment of the electron beam line.

\section{Experiment setup at nELBE and results}

The experimental program concentrates on inelastic neutron scattering and neutron induced fission cross sections measurements in the future. The time-of-flight of the neutrons is measured with plastic scintillators with a low detection threshold. A typical energy spectrum obtained from the time-offlight measurement is shown in Fig. 2. The experimental spectrum extends from $200 \mathrm{keV}$ to $10 \mathrm{MeV}$ peaking at around $2 \mathrm{MeV}$. The time resolution can be determined from the width of the photon peak (not shown). The full width at half maximum (FWHM) amounts to $750 \mathrm{ps}$. Electrons impinging on the lead circuit were simulated with MCNP and the resulting neutron spectrum was calculated, see Fig. 2. The high energy part of the spectrum seems to be underestimated slightly by the simulation, which might be due to the modeling of the preequilibrium emission.

The Data Acquisition system is VME-bus based using the MBS program from GSI, Darmstadt [6]. A high resolution multi-hit multi-event TDC CAEN V1190A is used for the time-of-flight measurements. The $\mathrm{BaF}_{2}$ detectors mentioned below are read out by dedicated TDC/QDC modules CAEN V874B. A flexible trigger logic is realized with CAEN V1495 FPGA module. The dead time per event of the multiparameter listmode acquisition is $70 \mu \mathrm{s}$.

Inelastic neutron scattering is being investigated using a compact $4 \pi$-array of $\mathrm{BaF}_{2}$ scintillators consisting of up to 42 hexagonally shaped crystals with length $20 \mathrm{~cm}$ and inner diameter $53 \mathrm{~mm}$ in combination with an array of 5 plastic scintillators of $100 \mathrm{~cm}$ length and $4.2 \mathrm{~cm}$ width and $1.1 \mathrm{~cm}$ thickness with low detection threshold [5]. Both detector types reach time resolutions clearly below $1 \mathrm{~ns}$ and are thus well suited for a proper time-of-flight tagging. Using the photon and neutron detectors in coincidence inelastic neutron scattering can be identified.

By a double time-of-flight technique the energy of the incoming neutron and of the scattered neutron can be determined. The plastic scintillators detect the scattered neutron and the $\mathrm{BaF}_{2}$ scintillators detect the deexcitation $\gamma$ ray. From the detection time of the $\gamma$ ray relative to the accelerator pulse

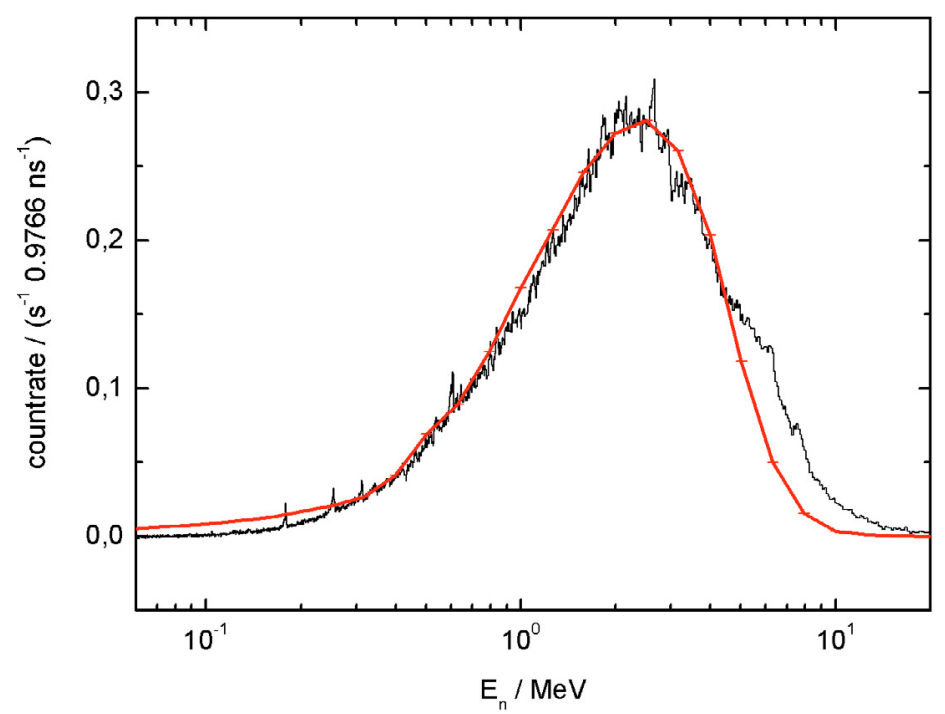

Fig. 2. Neutron energy spectrum measured at nELBE (black histogram). The electron beam energy was $33 \mathrm{MeV}$ and a flight path of ca. $652 \mathrm{~cm}$ was used. The red curve is an MCNP simulation. 


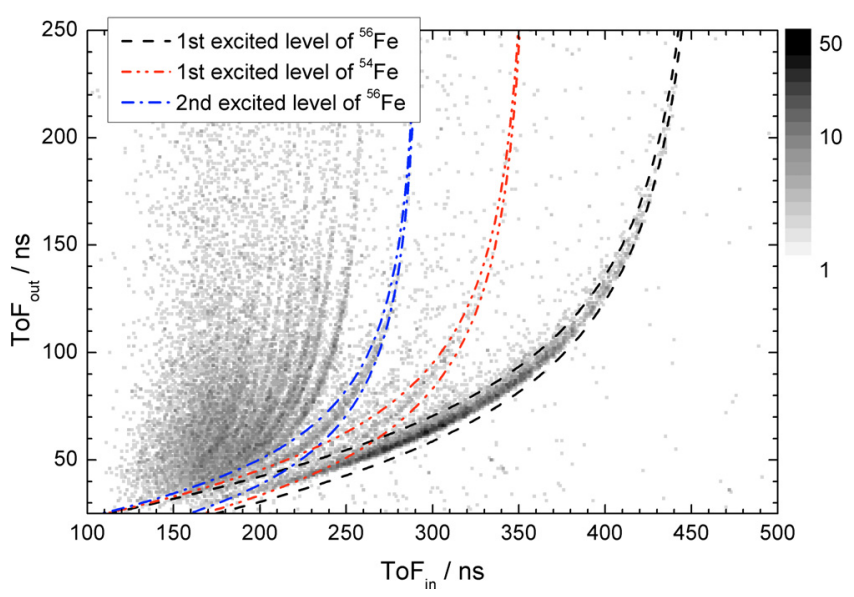

Fig. 3. Double-time-of-flight spectrum of ${ }^{n a t} \mathrm{Fe}$. The time-of-flight of the incoming neutron $\mathrm{ToF}_{\text {in }}$ is determined by the detection of the emitted $\gamma$-ray. The time-of-flight of the outgoing neutron $\mathrm{ToF}_{\text {out }}$ is determined by the time difference between neutron and $\gamma$ detection. The lines indicate the regions where the events from the inelastic scattering on ${ }^{55} \mathrm{Fe}$ and ${ }^{56} \mathrm{Fe}$ should appear.

structure one obtains the time-of-flight of the incoming neutron $\mathrm{ToF}_{\text {in }}$ from the source to the target. From the time difference between neutron and $\gamma$ detection one obtains the time-of-flight of the scattered neutron $\mathrm{ToF}_{\text {out }}$ from the target to the neutron detectors. In Fig. 3 the two-dimensional spectrum of neutrons scattered inelastically from a ${ }^{n a t} \mathrm{Fe}$ sample $(m=20 \mathrm{~g})$ is shown. Scattering from the lowest excited states of ${ }^{56} \mathrm{Fe}$ can be separated. Even the scattering from the 1 st excited state of ${ }^{55} \mathrm{Fe}$, which has an abundance of only $6 \%$, is visible. The background level in this spectrum in the regions where inelastic scattering events physically cannot occur is very close to the random background measured with an empty target. This was achieved using borated PE shielding that blocks scattered neutrons from the $\mathrm{BaF}_{2}$ detectors to reach the plastic scintillators. The neutron beam intensity was monitored by using a ${ }^{235} \mathrm{U}$ fission chamber from PTB, Braunschweig, to be able to perform absolute measurements. The neutron intensity on target was ca. $4 \cdot 10^{4} \mathrm{n} / \mathrm{s} / \mathrm{cm}^{2}$. The time resolution of the fission chamber was determined from the photo-fission peak width to be ca. 4 ns.

Using the energy distribution measured by the fission chamber and the events in the relevant region of the double time-of-flight spectrum one can calculate inelastic neutron scattering cross section for the level of interest. This is shown in Fig. 4 for the first excited level of ${ }^{56} \mathrm{Fe}\left(\mathrm{E}_{x}=847 \mathrm{keV}\right)$. A good agreement is reached with previous measurements by Perey et al. [8] up to the threshold of the second excited level at $2085 \mathrm{keV}$. There the method of measuring photon production yields only, has ambiguities, because the second excited level can decay via the first and thus produces the same $\gamma$-ray energy. The double time-of-flight method can easily distinguish different levels well above their thresholds (up to the point where the levels start to overlap due to of time-of-flight resolution), because the energy of the scattered neutron is determined. In Fig. 5 the relative uncertainty of the measurement performed at $\mathrm{nELBE}$ is shown. At about $\mathrm{E}_{n, \text { in }}=2 \mathrm{MeV}$, where the maximum of the neutron flux is, an uncertainty of about $8 \%$ was reached. In Table 1 the different contributions of the experimental uncertainty are listed. The main contribution comes from counting statistics which counts $\approx 5 \%$ at $2 \mathrm{MeV}$ and which increases at lower and higher energies due to the decreasing neutron flux. It willl be decreased by longer measurement and the use of the new superconducting RF injector [2] delivering a higher electron current resulting in an about ten times higher neutron flux. This will additionally decrease the uncertainty of the primary neutron flux. Corrections for multiple scattering in the target, scattering of neutrons on air and on the angular correlation of the emitted $\gamma$-ray and scattered neutron still have to be included.

The second important step to decrease the total uncertainty would be to decrease the systematic uncertainty of the neutron detection efficiency of the plastic scintillators. The efficiency of the unshielded plastic scintillators was measured with quasi monoenergetic neutrons at the PTB Braunschweig [5]. Five different neutron energies between 29 and $1202 \mathrm{keV}$ were used; the results of this measurement is 


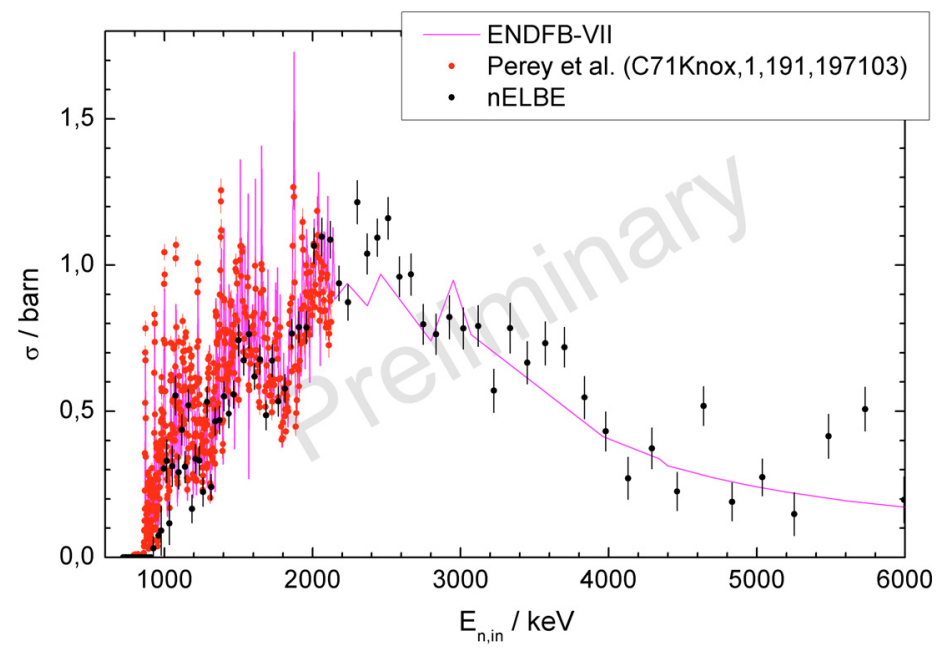

Fig. 4. The inelastic neutron scattering cross section ${ }^{56} \mathrm{Fe}$ measured at nELBE in comparison to previous measurements [8] and ENDFB-VII.0.

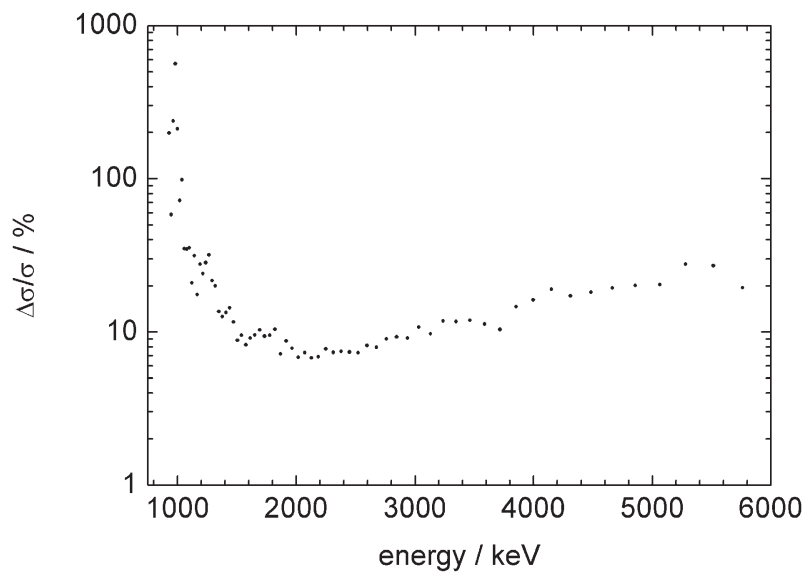

Fig. 5. Relative uncertainties of the measured cross section shown in Fig. 4.

Table 1. Total experimental uncertainty at $\mathrm{E}_{n, i n}=2 \mathrm{MeV}$.

\begin{tabular}{ll}
\hline Counting statistics (incl. empty target subtraction) & $\approx 5 \%$ \\
\hline Neutron primary intensity (Fission Chamber) & \\
Statistics & $\approx 3 \%$ \\
Efficiency & $\approx 3.8 \%$ \\
Geometrical scaling & $\approx 1 \%$ \\
\hline Detection Efficiency & \\
BaF $_{2}$ array & $\approx 1.5 \%$ \\
Plastic scintillators & $\approx 3.2 \%$ \\
\hline Total & $\approx 8 \%$ \\
\hline
\end{tabular}

shown in Fig. 6. In addition, the efficiency was measured in situ relative to the fission chamber to cover the whole energy spectrum. Both detectors were put in the nELBE neutron beam and measured the incoming flux at the same time. The result of this measurement is plotted in Fig. 6, too. A discrepancy to the PTB measurement can be seen at low energies, which might be due to fact, that the plastic scintillators are now surrounded by a $1 \mathrm{~cm}$ thick $\mathrm{Pb}$ shield. At higher energies structures from the neutron 


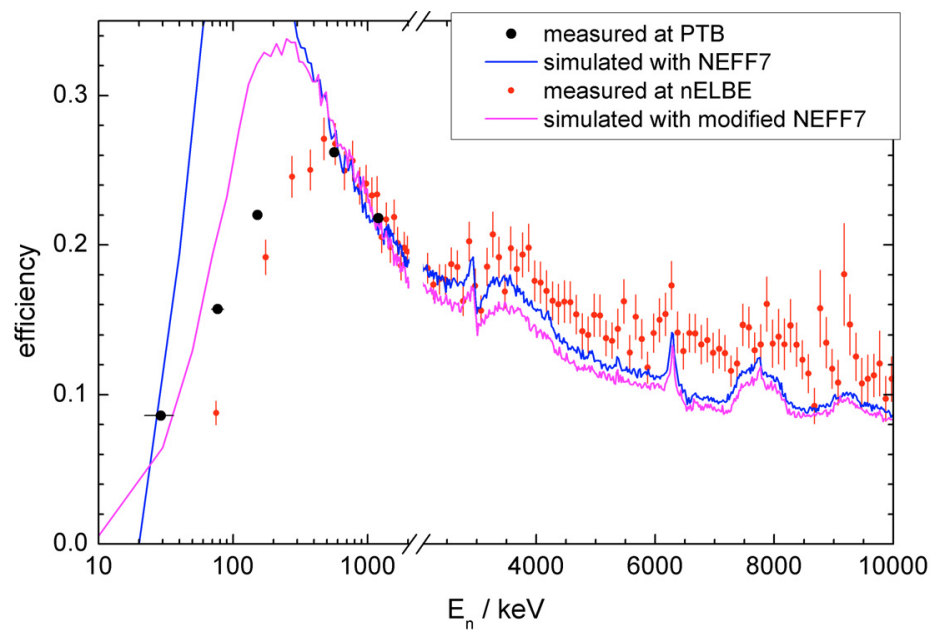

Fig. 6. Neutron detection efficiency of the plastic scintillation detectors.

interaction with the carbon in the scintillator are clearly visible. The structures were predicted by simulation calculations done using the code NEFF7 [10], a code well established for neutron efficiency simulation especially at energies above $1 \mathrm{MeV}$. Unfortunately NEFF7 cannot describe the region below $E_{n}<500 \mathrm{keV}$ here. Modifications taking into account the long detector geometry, the double-sided readout, wrapping of the scintillator, and PMT quantum efficiencies, could not completely reproduce the measured values (see Fig. 6). An experiment to re-measure the efficiency at the PTB is planned for the near future.

\section{Summary}

At the superconducting electron accelerator ELBE of the Forschungszentrum Dresden-Rossendorf the compact neutron time-of-flight facility nELBE has become operational. It is supposed to deliver data on neutron interactions in the energy range from $200 \mathrm{keV}$ to $10 \mathrm{MeV}$ which are important for nuclear transmutation facilities.

A setup for measuring neutron inelastic scattering cross sections by a double time-of-flight technique using $6 \mathrm{~m}$ and $1 \mathrm{~m}$ flight paths for the primary neutron beam and scattered neutrons respectively has been built. The $\gamma$-rays from inelastic scattering are detected with high time resolution in an array of $\mathrm{BaF}_{2}$ detectors and the scattered neutrons are detected in plastic scintillator panels with a low detection threshold. The neutron beam intensity is determined with a well characterized fission chamber from PTB. This method allows to determine absolute cross sections for single excited levels independently of the knowledge of level decay properties and thus can deliver complementary information to commonly used $\gamma$ production measurements.

Potential users are invited to submit proposals for experiments at nELBE.

nELBE is supported by the European Commission in the FP6-I3 project EFNUDAT (European Facilities for Nuclear Data measurements [9]) and by the German federal ministry of education and research (BMBF) under project number $02 \mathrm{NUK} 013 \mathrm{~A}$.

\section{References}

1. T.-Y. Song, S.-H. Park et al., Nuclear Data for Science and Tech. 2010, Jeju Island, ND1616.

2. R. Xiang, A. Arnold, H. Buettig et al., Phys. Rev. STAB 13, 043501 (2010).

3. E. Altstadt, C. Beckert, et al. Ann. Nucl. Ene., 34, 36 (2007). 
EFNUDAT Workshop “Measurements and Models of Nuclear Reactions", Paris, 2010

4. J. Klug, E. Altstadt, et al. Nucl. Instr. Meth. A, 577, 641 (2007).

5. R. Beyer, E. Grosse, et al. Nucl. Instr. Meth. A, 575, 449 (2007).

6. GSI Multi-Branch System, Ver. 2.2 (2000), http://daq.gsi.de.

7. e.g. http://www.nea.fr/janis/.

8. F.G. Perey, W.E. Kinney, and R.L. Macklin Proc. Int. Conf. Neutron Cross Sect. Tech., 191 (1971).

9. http://www.efnudat.eu.

10. G. Dietze and H. Klein, Report PTB-ND-22 (1982). 